\title{
LightCDD: Application of a Capability-Driven Development Method for Start-ups Development
}

\author{
Hasan $\operatorname{Koç}^{1^{*}}$, Marcela Ruiz ${ }^{2}$ and Sergio España ${ }^{2}$ \\ ${ }^{1}$ University of Rostock, 18051 Rostock, Germany \\ ${ }^{2}$ Utrecth University, Domplein 29, 3512 JE Utrecht, the Netherlands \\ hasan.koc@uni-rostock.de (orcid.org/0000-0002-1614-0230),m.ruiz@uu.n1 \\ (orcid.org/0000-0002-0592-1779), s.espana@uu.nl (orcid.org/0000-0001-7343-4270)
}

\begin{abstract}
Novice innovators and entrepreneurs face the risk of designing naive business models. In fact, lack of viability in business models is perceived to be a major threat for the start-up success. Both the literature and the responses we gathered from experts in incubation present evidences of this problem. The LightCDD method helps entrepreneurs in the analysis, design and specification of start-ups that are context aware and adaptive to contextual changes and evolution. In this article we describe the LightCDD method, a context-aware enterprise modeling method that is tailored for business model generation. The LightCDD applies a lightweight Capability-Driven Development (CDD) methodology. It reduces the set of modeling constructs and guidelines to facilitate its adoption by entrepreneurs, yet keeping it expressive enough for their purposes and, at the same time, compatible with the CDD methodology. We provide a booklet with the LightCDD method for start-ups development. The feasibility of the LightCDD method is validated by means of its application to one start-up development case. From a practitioner viewpoint (entrepreneurs and experts in incubation), it is important to provide integrative modeling perspectives to specify business ideas, but it is vital to keep it light. The LightCDD is giving a step forward in this direction. From a researcher point of view, the LightCDD booklet facilitates the application of LightCDD to different start-up development cases. The feasibility validation has produced important feedback for further empirical validation exercises in which is necessary to study the scalability and sensitivity of LightCDD.
\end{abstract}

Keywords: Capability-driven development, entrepreneurship, context-aware business model, start-up incubation, business model generation.

\section{Introduction}

By exploiting the niches and offering novel products and services, enterprising individuals with economic initiatives may create the resource for further employment. Hence, the

\footnotetext{
* Corresponding author
}

(C) 2017 Hasan Koç et al. This is an open access article licensed under the Creative Commons Attribution License (http://creativecommons.org/licenses/by/4.0).

Reference: H. Koç, M. Ruiz and S. España, "LightCDD: Application of a Capability-Driven Development Method for Start-ups Development," Complex Systems Informatics and Modeling Quarterly, CSIMQ, no. 10, pp. 53-74, 2017. Available: https://doi.org/10.7250/csimq.2017-10.04 
entrepreneurship and innovative activities is perceived to be a key factor in economic development [1]. However, the failure risks of start-ups are high and among the indicated reasons are the lack of viability in business models [2], [3], i.e. lack of realism (not enough examination of end user needs), and failing to envision contextual constraints (studying how the needs change over time), as shown in Figure 1. The lack of viability when modeling business ideas needs to be tackled by the enterprise modeling community. One way to bridge this gap could be the application of a modeling method that allows an integral view and supports different business perspectives, such as the needs of the entrepreneurs and end users, the sequence of activities to be followed to fulfil the needs, as well as the contextual factors influencing the procedures.
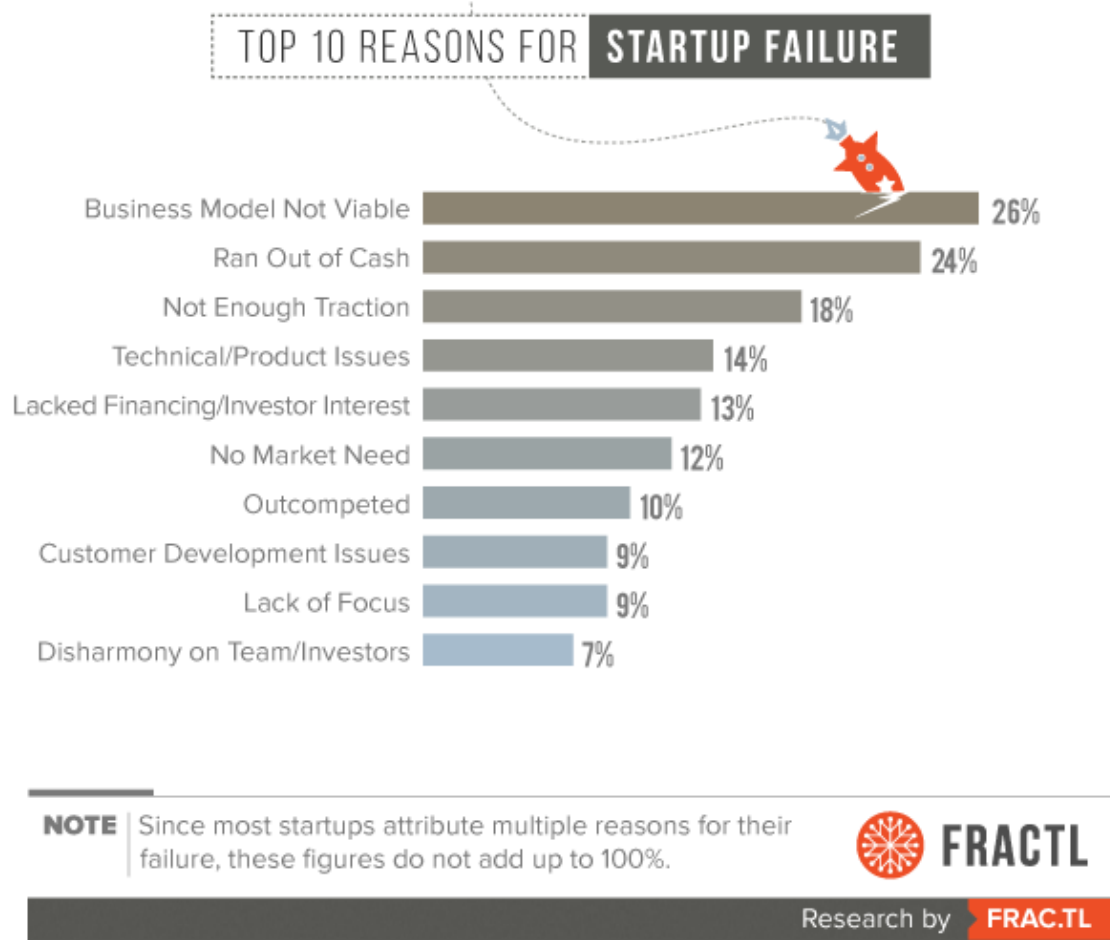

Figure 1. Top 10 failure reasons for start-ups [2], reproduced with permission

Recently, modeling and management of capabilities have been proposed as a way to cope with the challenges in dynamic environments. In that respect, a EU-FP7 project Capability as a Service in Digital Enterprises (CaaS) addresses methods and approaches with which the organisations may adapt to the changes and secure a competitive advantage [4]. To enable digital enterprises to sense and take advantage of changes in business context, CaaS engineers the Capability Driven Development (CDD) method. The CDD has been applied in various case studies for the evolution of information systems that need to incorporate context aware capabilities. Osterwalder et al. state that "a business model describes the rationale of how an organisation creates, delivers and captures value" [5]. Concerning the value creation part, we focus on the specification of business processes, goals, business capabilities, and contextual constraints of business environments. In this article we present the application of CDD for helping entrepreneurs in the analysis, design, and specification of start-ups. We explore CDD as a business tool that can complement current suites of business tools like the ones proposed in [5].

To shape our research, we conduct a design science project [6]. We perform the first iteration of a design cycle from the problem investigation to the treatment validation (see Figure 2). The first step is the problem investigation, which investigates the lack of modeling methods as a threat of start-ups shutdown. The second step corresponds to the treatment design, in which an exploratory exercise to apply CDD for entrepreneurship is performed. The third step corresponds 
to the treatment validation, which applies the CDD to two case studies of start-ups. Following the results, we conduct a design cycle in order to design a refined version of LightCDD. As part of the design, we specify a booklet to facilitate the application of LightCDD, which is validated by means of its application of the Let's Get Better case (one of the case studies). Several improvements of the LightCDD are then incorporated in the booklet as a result of the treatment validation (reprise of the task 5).

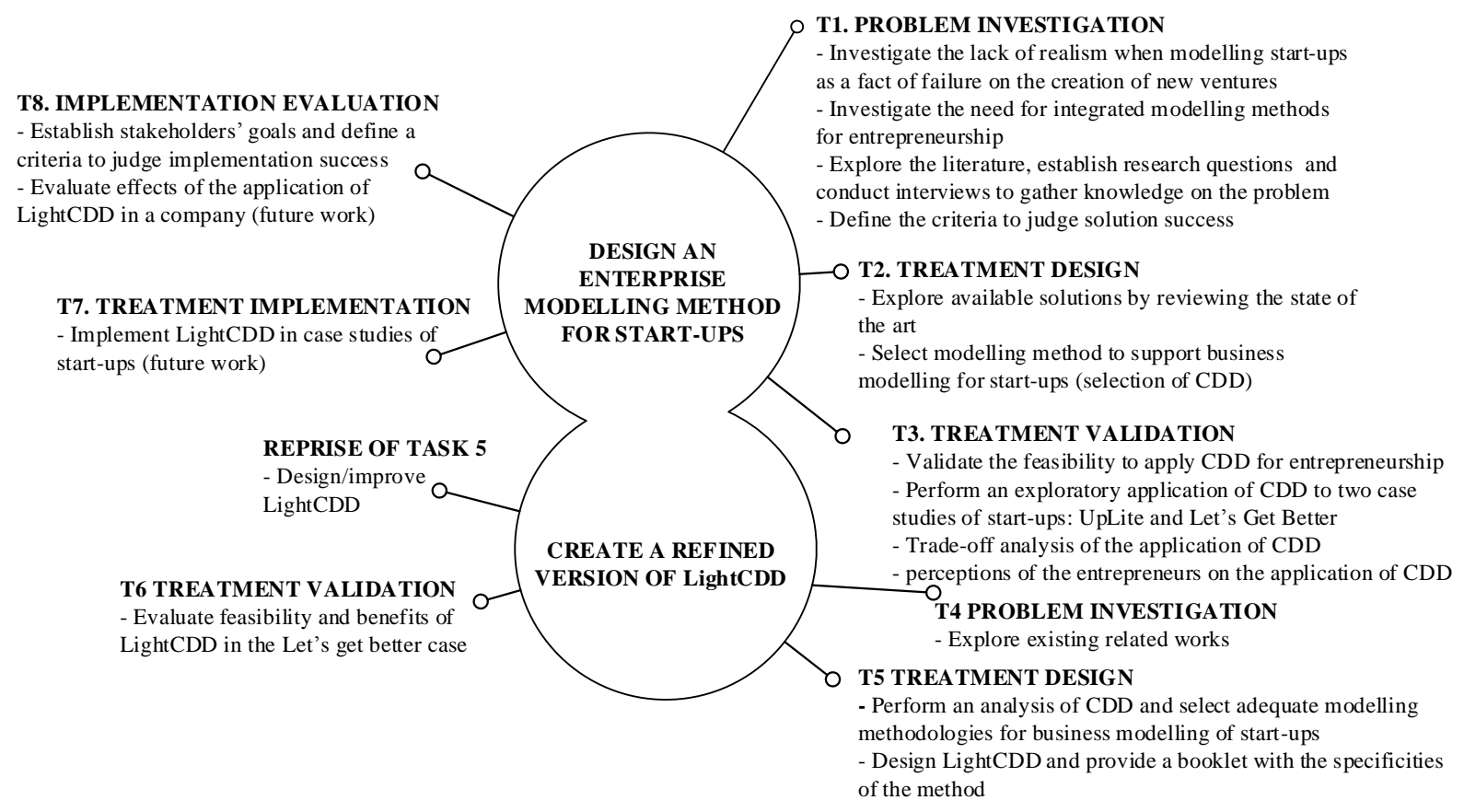

Figure 2. Overview of the research methodology

This article discusses the suitability of the application of CDD for start-ups, with a special emphasis on the information and communication technology (ICT) domain, i.e. whether it helps the entrepreneurs to reason on their business ideas and design. For this, we report on the application of the CDD in two exploratory case studies of start-ups, present the results of a survey regarding the method support for start-ups and then present the LightCDD, which is a simplified version of CDD aimed at the enterprise modeling activities of start-ups. We detail the LightCDD booklet in order to facilitate LightCDD adoption. The application of the LightCDD method is fully specified aiming at presenting the feasibility of the method.

The article is structured as follows. Section 2 discusses the main threats on start-up failures, outlines the related work, and presents the performed survey. Section 3 begins with the background information on the CDD paradigm, details its application in two case studies, and reports from the experiences gained during the application of CDD in practice, which motivates the LightCDD. Section 4 introduces the LightCDD, in this section the booklet to apply CDD is introduced. Section 5 presents an illustrative case to demonstrate the application of LightCDD in one start-up project, and Section 6 concludes the work.

\section{Problem Investigation}

The problem investigation is centred on the research about the lack of realism and the risks of not considering contextual and operative constraints for start-ups. We performed a literature review to investigate about the reasons of start-up failure. Also, we conducted a structured interview to investigate start-up failure reasons from the point of view of counsellors or managers of start-up incubators. 
Studies and analysis show that encouraging the entrepreneurship and innovative activities in small businesses is a key factor in economic development [7]. By exploiting the niches and offering novel products and services, enterprising individuals with economic initiatives sustain high levels of profitability, which may create the resource for further employment [8]. Based on this fact, the legislative bodies support the entrepreneurs to set up their own companies and consequently, the number of start-ups raise rapidly worldwide. However, the high start-up birth rates go hand in hand with a great risk of failure, it's estimated that 9 out of 10 start-ups fail [9]. As stated in [10] "the failure often surrounds five key dimensions: customers, business model, product, financials and team". Chorev and Anderson identify 8 top key topics and report that team commitment, team expertise, marketing, customer relationships, core team expertise and management, strategy, R\&D, and idea have high effect factors on start-ups success [11].

Among the various factors leading to the start-up failures, wrong estimations on the market need is one of the most important causes. Many start-ups focus too much on product, and not enough on examining the needs of the end user, which is prone to change rapidly. Another observed cause for failure is that the entrepreneurs underestimate the importance of planning, before entering the market [12]. Hence, due to the lack of realism in business ideas, many start-ups fail. To be more specific, different scenarios are neglected; the activities are captured in a single high-level process and the processes are not specified. Moreover, the objectives motivating the ideas and wishes are not captured, which should help to find a proper solution. Instead, the entrepreneurs rely on the subjective, and often biased, perceptions when creating a new business [13]. As such, business planning may help as a decisive factor for start-up success.

We have asked two experts in enterprise incubation to answer our questions on the reasons for start-up failure ${ }^{\dagger}$. The respondents are the manager of a start-up school (pre-accelerator), and the manager of an incubator who is also a lecturer of an ICT Entrepreneurship course in Utrecht University. The managers have 3 and 10 years of experience running incubators, respectively. Text box 1 elaborates on the findings.

Typical entrepreneurship cycles involve to some extent the following activities: generating and filtering ideas, modeling the business, planning the marketing and sales, validating assumptions, aligning resources and financing, and eventually establishing the enterprise. The amount of documentation depends on the overall approach to entrepreneurship, ranging from detailed business plans, which serve the purpose of specifying the ideas and serving as a means of communication with other stakeholders (e.g. potential partners, funders), to itemised templates that facilitate a lean. According the interviewees (and our own) experience, many unrealistic assumptions are made during business modeling; also during marketing, pricing and financial estimations, novel entrepreneurs are prone to overestimate, underestimate or overlook relevant factors. There exist several methods that help entrepreneurs conceive the factors that are key to the business success. The Business Model Canvas is a template used to structure aspects related to the value proposition, partners, infrastructure, customers, and finances [5]. The Lean Canvas [14], the Strongly Sustainable Business Model Canvas [15], and the Board of Innovation business model kit [16] are similar in their approach. Their popularity lay in the fact that they are a lightweight means to brainstorm, document, and communicate the elements that build up the business idea and factors expected to influence its success. To some extent, these business modeling methods are comparable to LightCDD; the difference strives in the fact that LightCDD focuses on the contextual factors and their influence on business processes. Furthermore, different from prevalent methods, LightCDD provides entrepreneurs with an appropriate notation, a number of templates and an open source software to sketch and elaborate their business ideas. It also adopts a capability-oriented view, which should support the start-ups in the construction of the minimum viable product and aligning its prerequisites with the entrepreneurial objectives.

\footnotetext{
${ }^{\dagger}$ The questions can be found at http://bit.ly/2kKBXoh
} 
Text box 1. Reasons of start-up failure

\section{Factors that influence start-up failure}

Entrepreneur goals and characteristics.

- Most of people that are part of start-ups creation are not entrepreneurs. People belonging to start-ups might be smart and have vision statement, but "entrepreneurship is a trait".

- "Entrepreneurs are not addicted to cash", they do not envision how to profit from their business ideas. For entrepreneurs it is easy to preach their ideas, but later they are not eager to work on them.
Entrepreneur environment.

- It is difficult to set-up a team to run a start-up; a poor team is a high risk for start-up failure.

- There are too many job prospects in ICT, most of the start-ups just shutdown due to teams get job offers that are more attractive than pursuing the success of a venture.

- Start-ups are set-up "at the wrong time in the wrong place".

The behaviour of young entrepreneurs and their lack of realism when modeling business ideas

By young entrepreneurs we refer to entrepreneurs without experience for designing start-ups. The interviewees somewhat agree on the idea that entrepreneurs tend to oversimplify the key activities when designing business processes. Nevertheless, they argue "no entrepreneurs would go through the creation of a new enterprise if they knew how hard it is".

On the other hand, the interviewees completely disagree with our hypothesis that "the younger the entrepreneur is, the more naive s/he tends to be while conceiving business ideas". It is clear that practice makes the master, but some entrepreneurs during her/his first attempt to set-up a company get successful.

\section{Lack of realism of business ideas}

The interviewees agree on the fact that entrepreneurs are more focused in the design than in the analysis during the development process of their ventures. This lack of proportion is a cause of failure. On the other hand, opinions of the interviewees are opposite regarding to how realistic are entrepreneur initial business ideas.

\section{Methods and instruments to support entrepreneurship}

Interviewees perceive that existing methods and instruments are appropriate to think about contextual constraints of the real world, and then entrepreneurs come up with realistic business plans.

Perceptions are divided when analysing current methods. One of the interviewees perceives that current methods do not allow the entrepreneurs have an integral view of their goals, activities, and contextual constraints. For that reason, providing mechanisms to represent contextual constraints and to reason about goals and processes would help entrepreneurs to anticipate and mitigate risks. Both of the interviewees agree on methods that provide separate specifications of enterprise models could help entrepreneurs to understand their business context. In addition, one interviewee indicates that modeling methods for entrepreneurs should be "graphical, technical, and lightweight. Different perspectives should be possible". Regarding to the needed perspectives for modeling business ideas, they mention that it is important to provide support for several complementary perspectives, like the ones offered by the CDD methodology, but they claim on the need to "keep it simple". Despite the fact modeling is important for entrepreneurs, one of the interviewees commented that "it is the art of entrepreneurship to not get trapped in analysis paralysis and still having sufficient insight to take the right decisions".

To conclude, we found various evidences on the need to provide lightweight and integrative modeling supports for start-ups. In the following sections we explore the applicability of CDD for entrepreneurship support and start-up modeling.

\section{First Experiences on Applying CDD in Start-ups}

\subsection{Capability as a Service in a Nutshell}

CaaS project aims to create an integrated approach consisting of a method, tools, and best practices that enable digital enterprises to sense and take advantage of changes in business context. The CaaS methodology for capability-driven development (CDD) consists of various components addressing different modeling aspects, such as context modeling, business process modeling, pattern modeling, and adjustments modeling. The method is supported by the CDD environment, which comprises of a Capability Design Tool (CDT) incorporating a context modeling module, a Capability Context Platform (CCP) to monitor the contextual values at runtime as well as a Capability Delivery Navigation Application (CNA), which enables adjustments in line with the service delivery context and reusable best practices [4]. 
Three use cases in the sectors of e-government [4], energy [17], and insurance [18] prove that the methodology is successful when applied to full-fledged enterprises that intend to become more context-aware and adaptive. However, studies on the application of the CDD and its effects in innovative start-up projects in entrepreneurial settings are missing.

\subsection{Two Practical Start-up Cases}

Digital technology powered by the growth in the digital economy caused an increase in the number of young entrepreneurs and innovative ideas. This specifically can be observed between the two main sectors, namely, telecommunications and ICT [8]. We have launched the yes!PoEM ${ }^{\ddagger}$ seminar for entrepreneurs, in which the DELITELABS ${ }^{\S}$ start-up school has participated. This section focuses on the two innovative start-ups in the ICT domain for which we apply the CDD paradigm ${ }^{* *}$, they are summarised in the Text box 2 .

\section{Text box 2. Start-up Cases}

\begin{abstract}
Case 1: Let's Get Better
Let's Get Better is an online platform that provides users with a health coach in their pocket. The main motivation of the project is that an active and healthy life is the key to a wellbalanced life but the people struggle adopting healthy habits, since they are busy with their work and social life. As a solution, the platform takes advantage of digital technology and offers 1:1 professional coaching through instant messaging and weekly video calls, progress tracking and peerto-peer networks. To further support and encourage users, a social media will allow people to share their story, gain further support, and build common-interest relationships.
\end{abstract}

\section{Case 2: UpLite}

UpLite is a startup project, where the entrepreneurs propose new way of charging smartphones by improving social contacts. The motivation behind the project is that difficulties arise when one looses the online connectivity, i.e. people feel lost and unsecure when these devices run out of battery and they are limited in terms of their reaching their contacts and information supply. As a solution, the digital entrepreneurs produced a hardware, which connects two mobile devices, allowing them to exchange battery life. In addition to that, they developed a mobile application, which supports such exchange by showing the energy providers and their locations on a map. In this way, the mobile device user with less battery-life can look for the best options near him and after finding the provider, the device gets charged by the other device. The additional value lays in the social interaction with the energysharing companion while charging the smartphone.

The entrepreneurs follow two optional business models, where they do not directly charge the users. The first option is to collaborate with a big marketing campaign of a large consumer brand, such as Starbucks, T-mobile or Coca-Cola, which increases the possibilities of a quick scaling up. The second option is to partner up with bars and restaurants and charging them a monthly fee per month per hardware device that is provided to them. In return, the bars and restaurants would be advertised on the map in the application.

\footnotetext{
${ }^{*}$ http://www.pros2.webs.upv.es/en/yespoem

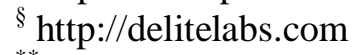

** The posters with the projects' descriptions can be found at http://bit.ly/2jpTo0hPOSTER1 and http://bit.ly/2kmAlncPOSTER2
} 


\subsection{Applying the CDD}

The CDD supports different entry points and offers three strategies for capability modeling, namely, goal-based, process-based, and concept-based approaches [19]. The method that describes the application of CDD to the two cases (cf. Text box 2) is depicted in Figure 3, using the Process Deliverable Diagram notation [25].

Before starting the modeling sessions, the authors explained the teams the CDD concepts in detail, i.e. the terms business processes, context, and capability were defined. After that, both the Let's Get Better team and the UpLite team were asked to provide us with the business processes (see activity 2 in Figure 3). The results were generic and did not quite reflect the actual implementation of their business ideas.

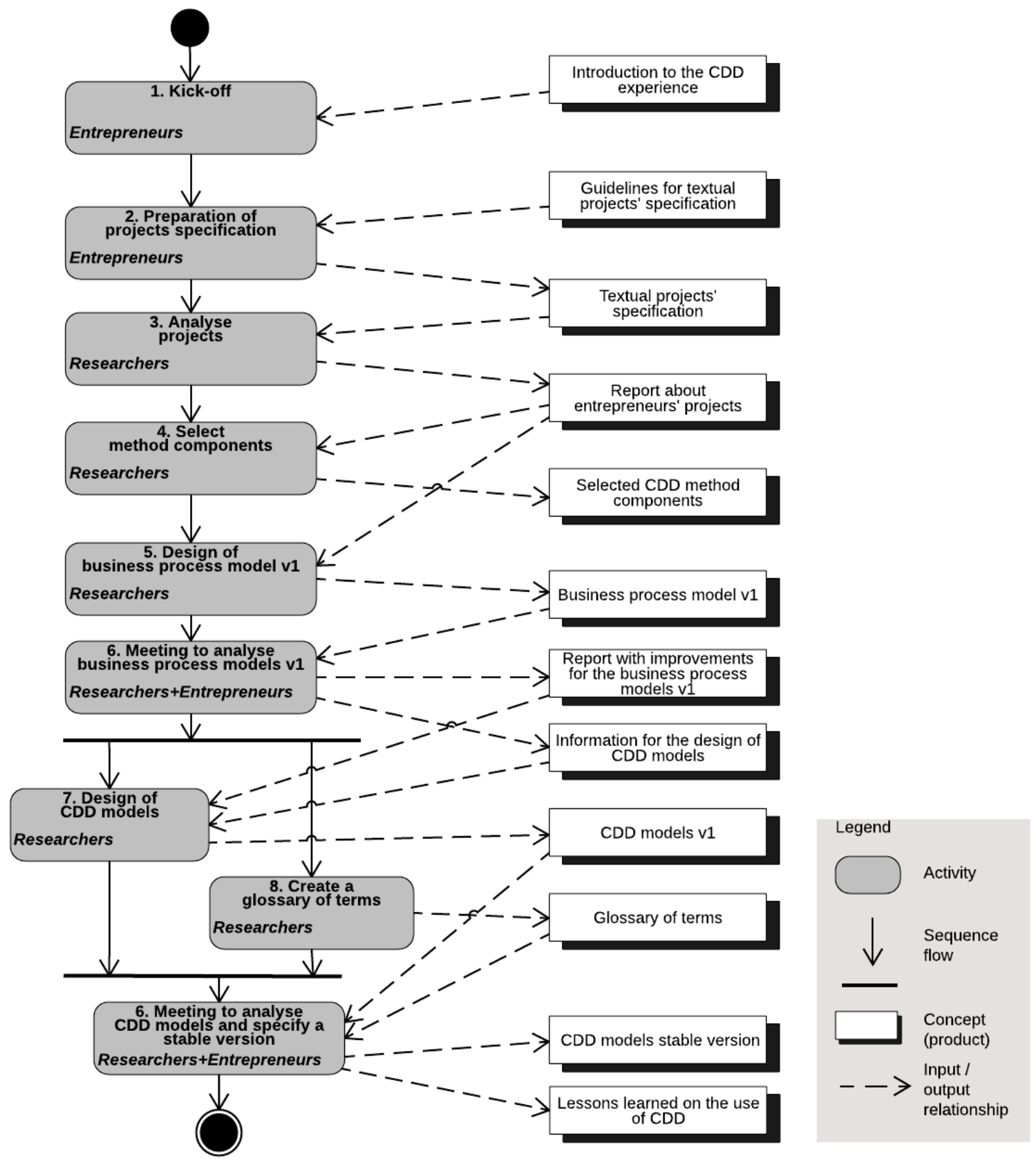

Figure 3. CDD application procedure for the two start-up cases 
CDD allows for different strategies when modeling capabilities [19]. To select the right strategy, we analysed the qualitative data provided by the entrepreneurs (see procedure 3 in Figure 3). The data included the descriptions of the use cases as well as the explanations of additional concepts, key activities, resources, and stakeholders. Following that, we met with the teams in an interactive group setting. Based on the gathered information, we identified that the goals were not reflected at all or only expressed ambiguously. Regarding the organizational structures, both teams had clearly defined roles and responsibilities. However, in terms of key concepts, the teams lacked a shared understanding, thus further work was needed to clarify such concepts. In this respect, the teams received an additional task to create a glossary of terms before starting with the capability modeling sessions. In contrast, we identified that in both use cases the teams possessed knowledge on the key activities. Consequently, the process-first capability design strategy seemed to be the most suitable one.

Using the CDD method, first the business process models were updated ${ }^{\dagger \dagger}$. In the next step, we identified the core capabilities and, together with the teams, we prioritized them and selected one capability per use case. Following that, the goals of the team alongside with the end user needs were analysed and modeled, these were related to the capability and to the business process models. Finally, based on the gathered information, the contextual factors possibly effecting the service provision were explicated and modeled. In each iteration, additional context elements were identified and their effect on the business idea were discussed. The results were used as an input for a new interaction with the entrepreneurs, i.e. the steps regarding the modeling of the business processes and goals were revisited (see procedure 9 in Figure 3).

\subsection{Lessons Learned, Experiences}

Looking back at the success factors as introduced in Section 2, the application of the CDD led to a more committed team with a shared understanding and clearly defined objectives. In particular, the modeling activities enhanced the communication between the core team. The developed models contributed to an overview of the core product or service and how to offer it, the management had useful instruments to define their strategy. Moreover, goal models reflected not only the objectives of the start-ups, but also of the potential customers, which is expected to improve the customer relationships, probably opening new marketing channels. The whole CDD practice helped to enrich the business idea and minimize the risk of a false start.

Concerning the causes of the failure, the CDD improved the start-ups in the identification of the real world factors in the business. To exemplify, the UpLite team did not consider the context of the user in different scenarios, so their app did not incorporate the required functionalities, such as the user profiles, willingness to share, ratings from other users, distance to energy providers and battery status. For the Let's Get Better Team, the capability model provided them with the information they needed to start developing an algorithm for the app, which was not considered before.

In line with the findings from the literature, another important failure factor was that the entrepreneurs tend to design simple processes. In both cases, the procedures were vaguely defined and many gateways were not considered explicitly. After applying the CDD, the core business process models are updated. Each meeting with the entrepreneurs resulted in a more mature process model and addressed additional discussion points for the teams in terms of a concrete implementation of the activities.

We interviewed the CEOs of the two cases that have applied CDD. Both of them did not have previous experience with enterprise modeling before the application of the CDD. In Text box 3 we present the research questions that stand for the structure of the interview, and the insights providing answers to the research questions.

\footnotetext{
${ }^{\dagger}$ The respective models before and after CDD application can be found at http://bit.ly/2kbTGVW
} 
Text box 3. Interview questions and responses

(RQ1) What is the perceived usability and ease of use of CDD in start-ups projects?

- It was easy to apply and is useful to understand and design start-up projects. One CEO says, "CDD is a logical process, which helps to provide structure in a wildly abstract start-up". Nevertheless, the first impression that the entrepreneurs got from CDD was the uncertainty on how CDD could be applied to their projects.

- Regarding the CDD's concepts: “The terminology used is still rather confusing though". The CDD's concepts need to be reviewed and tailored for end-users.

(RQ2) What is the perceived impact after the use of CDD in start-ups projects?

- CDD would increase the chances that a start-up achieves its business goals in a changing environment.

Although, one comment stands for the idea that CDD seems to be ICT project-dependent.

- CDD helps identifying gaps in business plans. But, for future use of CDD, one CEO states that: "If I feel like there's a need to methodically define a process, then yes - definitely".

- From the benefits point of view, the "CDD helped on structuring and clarifying the process" and "The business process model provided us with a clearer user journey. The capacity model provided us with the information we needed to start developing an algorithm for the app".

In general, CDD helped them to find out further configurations of their products and services by analysing their business ideas from the capability point of view. The entrepreneurs identified gaps in the distribution and revenue models, which were optimized (cf. Text box 3: RQ2). Nevertheless, applying the CDD in full had a few drawbacks. First, the entrepreneurs were initially unsure on how to apply CDD to their projects, which is mainly related to the number of method components. The CDD focuses on the application scenarios of mature enterprises that intend to become context-aware and adaptive, which necessarily requires engineering of a comprehensive method with a number of components. For the two cases, the CDD was not applied in full and just some method components were selected for their application. Second, the method terminology was rather confusing for them and needed review. The teams were not able to apply the CDD by themselves, which created a complicated CDD application procedure, the intervention of the researchers to the modeling processes was required, and the created models are analysed with the entrepreneurs (cf. Figure 3). To overcome these drawbacks, we propose the LightCDD, which balances the trade-off between the simplicity and expressiveness.

\section{LightCDD Method: a Booklet}

As a result of the application of CDD in two start-up projects, we found that it is feasible to apply CDD for start-ups projects. As we presented in Section 2, all respondents of the questionnaire agreed that the entrepreneurs are more focused on the design than on the analysis during the development process of their ventures; and this lack of proportion is a cause of failure. Thus, methods to analyse their ideas should be provided to the entrepreneurs. Regarding the characteristics of such methods, they should support graphical representation of the business idea, be lightweight and simple. Moreover, it is required to provide mechanisms to represent contextual constraints that help entrepreneurs anticipate and mitigate risks of failure, and provide integrative view of goals, activities, and contextual constraints.

Taking into account the interview responses (cf. Section 3.4), we propose the LightCDD, which is a simplified version of CDD with a different purpose, i.e. not providing support in a full-fledged company, but rather in a start-up design. To meet the demands of the start-ups, the LightCDD allows for a holistic view on the business idea by incorporating the objectives to fulfill (goals view), the activities implemented to realize the business idea (process view), and change factors affecting the implementation of the business idea (context view). LightCDD aims to fulfill the following objectives:

- Provide systematic support to specify the models of a business idea based on enterprise modeling techniques. 
- A capability-based assessment of a business idea, i.e. examination of how viable it is in dynamic environments and identification of potential gaps.

- Thorough documentation of the activities required to implement a business idea as well as their contextual adaptation to changing situations.

- Elicitation of the team objectives in form of SMARTly expressed goals to investigate the end user and market needs.

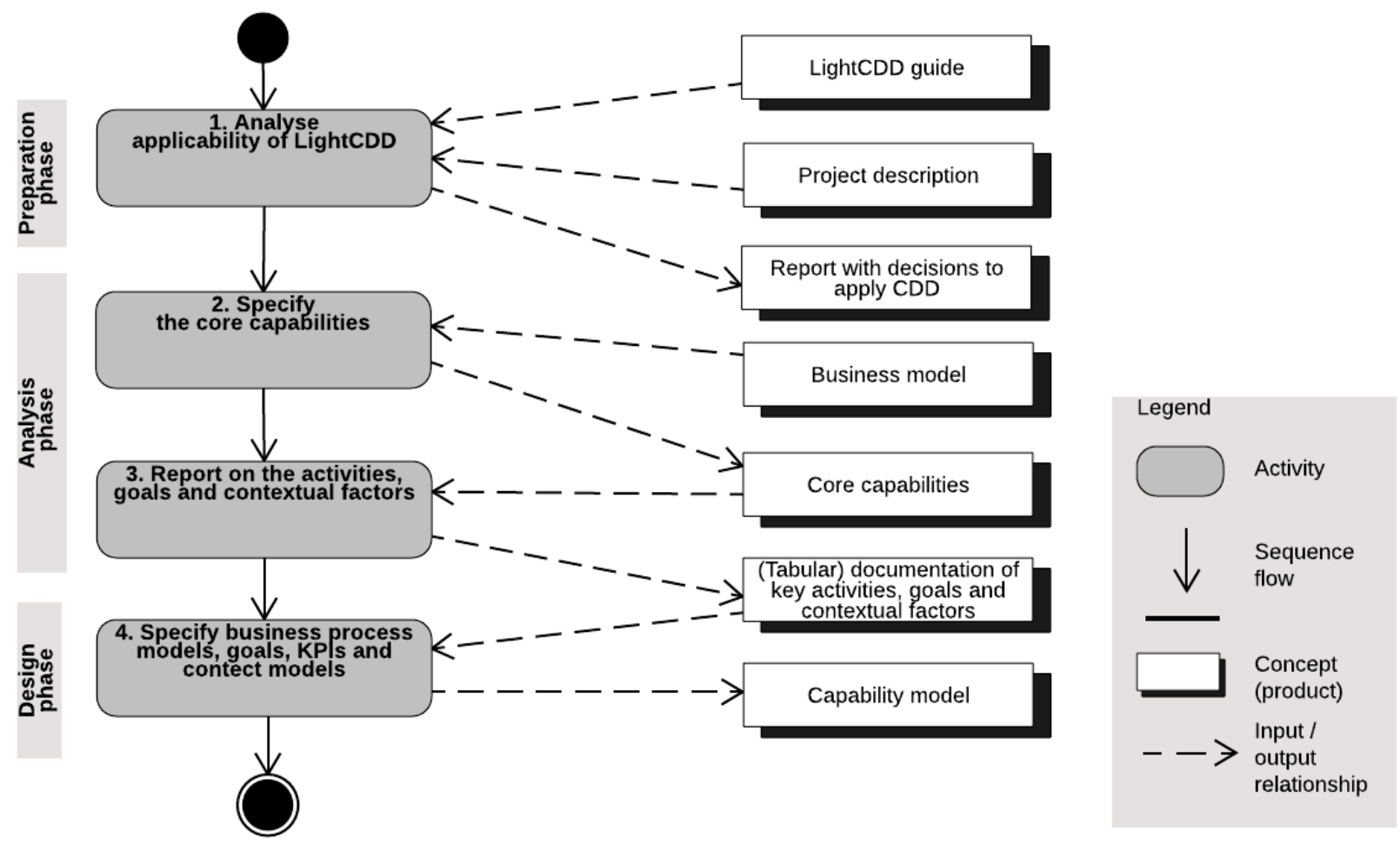

Figure 4. Overview of the LightCDD

LightCDD consists of three phases, namely, preparation, analysis, and design. Figure 4 illustrates the activities of each phase as well as the inputs required and outputs produced by those activities, again using the Process Deliverable Diagram notation. The preparation phase checks the applicability of the method and helps the user to understand the main concepts and benefits of LightCDD. Application of this part is mandatory. Afterwards, the entrepreneurs are recommended to proceed with the analysis phase. Depending on the level of knowledge in conceptual modeling, the method user can also apply analysis and design phases in parallel. Each phase is described in detail in Sections 4.1, 4.2, and 4.3 respectively. The notation required in the design phase is introduced in Section 4.4.

\subsection{Preparation Phase}

In this phase, introductory information is given to the method users, i.e. which enterprise modeling techniques are used in the method and what are the typical application scenarios.

It is recommended to apply the LightCDD, if one or more of the following statements hold true [20]:

- If one plans to offer the same business for different target groups and markets.

- If there are variations in the execution of business ideas or certain situations for various application scenarios.

- If a business idea is closely related to digitalisation and delivers ICT-based solutions. 
- If one has not used a proprietary development methodology that supports the system development life-cycle thoroughly.

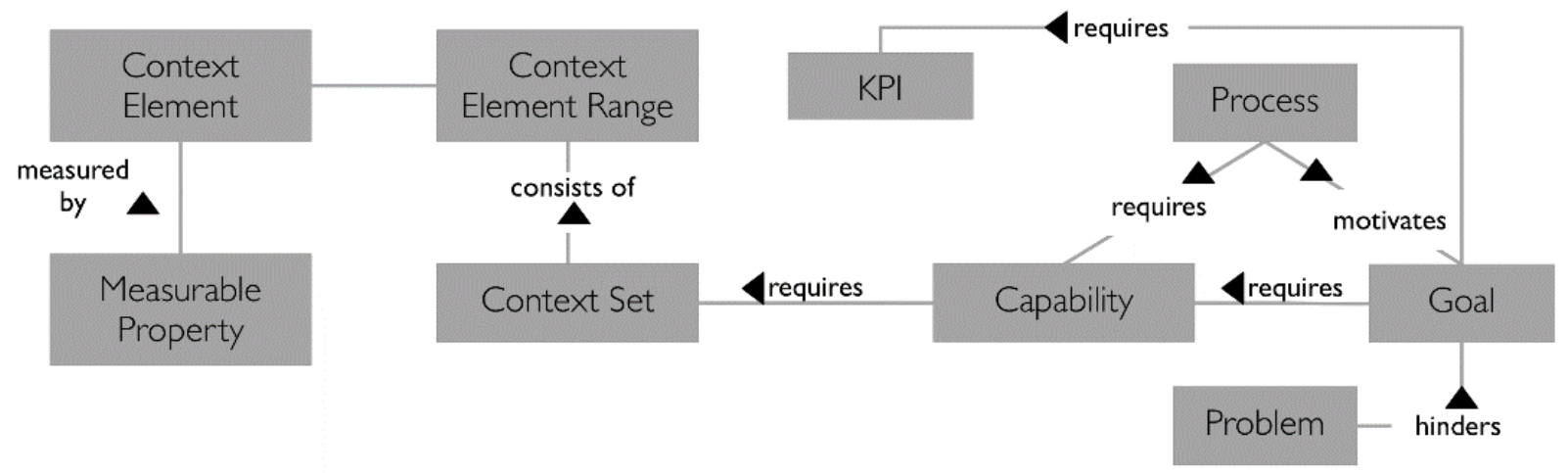

Figure 5. LightCDD meta-model

Table 1. Important Concepts and their Definitions

\begin{tabular}{|c|c|c|}
\hline Concept & Definition & Example \\
\hline Capability & $\begin{array}{l}\text { Ability and capacity that enable start-ups to achieve } \\
\text { entrepreneurial objectives under changing environments. }\end{array}$ & $\begin{array}{l}\text { Battery life exchange based } \\
\text { on a social interaction }\end{array}$ \\
\hline Goal & $\begin{array}{l}\text { Desired state of affairs that has to be attained [4]. Goals } \\
\text { should typically be expressed in measurable terms such as } \\
\text { KPIs. }\end{array}$ & $\begin{array}{l}\text { To allow an exchange of } \\
\text { battery life between the most } \\
\text { near devices or users }\end{array}$ \\
\hline Problem & $\begin{array}{l}\text { A gap between the current state and a desirable state as } \\
\text { perceived by the participants in practice [21]. }\end{array}$ & $\begin{array}{l}\text { People feel unsecure when } \\
\text { their devices run out of } \\
\text { battery }\end{array}$ \\
\hline Process & $\begin{array}{l}\text { Series of actions that are performed in order to achieve } \\
\text { particular result. A Process supports Goals and has input and } \\
\text { produces output in terms of information and/or material. } \\
\text { When initiated a process is perceived to consume resources. }\end{array}$ & $\begin{array}{l}\text { Capture user profile and } \\
\text { preferences }->\text { Run the } \\
\text { algorithm }->\text { Rate the UpLite } \\
\text { user }\end{array}$ \\
\hline KPI & $\begin{array}{l}\text { Measurable properties that can be seen as targets for } \\
\text { achievement of Goals. }\end{array}$ & $\begin{array}{l}\text { Number of users that } \\
\text { exchange energies }\end{array}$ \\
\hline $\begin{array}{l}\text { Context } \\
\text { Element }\end{array}$ & $\begin{array}{l}\text { Dynamic entities that affect the design and provision of the } \\
\text { core business idea. Managing context entities is crucial to } \\
\text { fulfil entrepreneurial objectives. }\end{array}$ & Battery status \\
\hline $\begin{array}{l}\text { Context } \\
\text { Element } \\
\text { Range }\end{array}$ & Permitted values for a specific context element. & Low, Mid, High \\
\hline $\begin{array}{l}\text { Measurable } \\
\text { Property }\end{array}$ & Attribute of a context element that help to measure its value & Battery charge rate \\
\hline Context Set & $\begin{array}{l}\text { A container for context elements that are relevant for design } \\
\text { and delivery of a specific capability. This container includes } \\
\text { defined value ranges of a context element and is related with } \\
\text { the capability. }\end{array}$ & $\begin{array}{l}\text { Collection of the context } \\
\text { elements battery status and } \\
\text { user socialness, related with } \\
\text { aforementioned capability }\end{array}$ \\
\hline
\end{tabular}

LightCDD uses enterprise modeling techniques to support the start-ups. For this purpose, the method uses goal modeling, process modeling, and context modeling in particular.

- A goal model illustrates the objectives of the start-up and the typical problems that might occur in fulfilling these. It also helps to assess the end user needs in the market.

- A business process model represents the realization of the business idea, i.e. it documents the procedures (workflows, activities, steps) that has to be implemented to fulfill the goals.

- Business processes are strongly influenced by the environmental factors. The real implementation of a business process requires selecting the right activities with right orders, which strongly depends on the application scenario. Context models capture and document 
such scenarios, relate them with the objectives and capabilities of the start-up with the aim of increasing the viability of the business idea also under changing conditions.

To introduce the important concepts used by the LightCDD, we use a simplified version of the meta-model developed in the CaaS project [4]. The relevant aspects of reality or, in other words, important concepts and the "language" of the LightCDD, are depicted in Figure 5 and explained in Table 1. To enhance the understanding of the concepts, we use examples from the UpLite case, which is described in Section 3.2 (cf. Text box 2).

The output of the preparation phase should be a report regarding the LightCDD applicability. The method's user should adopt the template shown in Table 2 and provide answers for documenting the decision concerning the method's application.

Table 2. Template to produce the output of the first phase

\begin{tabular}{|l|l|}
\hline \multicolumn{1}{|c|}{ Questions } & \multicolumn{1}{c|}{ Answers } \\
\hline $\begin{array}{l}\text { Do you plan to offer the same business for } \\
\text { different target groups and markets? }\end{array}$ & <Answer about the business strategy> \\
\hline $\begin{array}{l}\text { Are there variations in the execution of business } \\
\text { ideas or certain situations for various application } \\
\text { scenarios? }\end{array}$ & $\begin{array}{l}\text { <Answer about potential contextual variations and } \\
\text { change> }\end{array}$ \\
\hline $\begin{array}{l}\text { Is your business idea closely related to } \\
\text { digitalization and deliver ICT-based solutions? }\end{array}$ & $\begin{array}{l}\text { <Answer about a potential implementation of the } \\
\text { business idea by means of ICT services. This indicates a } \\
\text { further use of model-driven development for } \\
\text { information systems> }\end{array}$ \\
\hline $\begin{array}{l}\text { Have you used a proprietary development } \\
\text { methodology that supports your system } \\
\text { development life-cycle thoroughly? }\end{array}$ & $\begin{array}{l}\text { <Answer about the state of the project and potential } \\
\text { integration with current methodologies for business } \\
\text { specification and development> }\end{array}$ \\
\hline
\end{tabular}

\subsection{Analysis Phase}

In the analysis phase, the LightCDD recommends to apply a participatory approach, which refers to the practice of incorporating stakeholders into the elicitation process [22]. Consequently, the entrepreneurs that conceive the business idea and play a role in its implementation should be involved in the analysis phase. The following steps are recommended by the method:

- Focus on the principles of your business idea and on the main proposition, specify the core capability. To do so, you can prepare a list of all offers or implementation scenarios that have variations in the way they are performed depending on certain conditions. Your capability must satisfy all such scenarios and situations.

- List the goals that should be fulfilled by implementing the business idea. The answers to the following questions will help the entrepreneurs to identify, structure, and prioritize goals.

- What do you want to achieve or avoid by implementing the business idea? What problems hinder achievement of such goals?

- Which strategy does your start-up follow? Which partners exist and what are the available key resources?

- How is the value proposition distributed?

- Enumerate and list all key activities that pertain to the selected capability, i.e. should be executed to realize the business idea. 
- Analyze the contextual factors influencing the key activities. To do so, the LightCDD proposes the following guidelines:

- Investigate the conditions that cause the implementation of the key activities in various ways (e.g. change in the value proposition in a non-EU market). Elicit the factors that may influence the execution of the business idea (e.g. regulatory changes).

- List the factors and prioritize them depending on their effect on goal fulfillment.

Each iteration may require altering the core capability which is defined in the first activity. The output of the analysis phase is a tabular documentation of key activities, goals, and contextual factors. For this purpose, LightCDD supports the method user with three templates, which are shown in Table 3, Table 4, and Table 5 respectively ${ }^{\text {ț }}$.

Table 3. Template for documenting problems and goals

\begin{tabular}{|l|l|}
\hline \multicolumn{1}{|c|}{ Problems/ objectives } & \multicolumn{1}{c|}{ Description } \\
\hline Problem 1 & $<$ Description of the problem $>$ \\
\hline Goal 1 & $<$ Description of the goal $>$ \\
\hline$\cdots$ & $\cdots$ \\
\hline
\end{tabular}

Table 4. Template for documenting key activities

\begin{tabular}{|l|l|}
\hline \multicolumn{1}{|c|}{ Activity } & \multicolumn{1}{c|}{ Description } \\
\hline A1. & $<$ Description of the key activity or related business processes $>$ \\
\hline$\cdots$ & $\cdots$ \\
\hline
\end{tabular}

Table 5. Template for documenting contextual factors

\begin{tabular}{|l|l|}
\hline \multicolumn{1}{|c|}{ Activity } & \multicolumn{1}{c|}{ Description } \\
\hline C1. & $<$ Description of the contextual factor $>$ \\
\hline$\cdots$ & $\cdots$ \\
\hline
\end{tabular}

\subsection{Design Phase}

In this phase, the textual input produced during the analysis phase will be represented graphically. For this purpose, the LightCDD notation will be used, which is documented in Section 4.4. The following procedures are recommended:

- Test, first model the capability or capabilities defined in the analysis phase.

- Capture the objectives and the problems that should be solved by implementing the business idea in goal models (cf. Section 4.4).

- Add KPIs to the goals (can also be done in the further iterations).

- Model the key activities as business processes by using the Business Process Model and Notation (BPMN) [23].

- Model the contextual factors (context element), how they are measured (measurable properties) and their valid ranges (context element ranges). As a capability requires one and only one context set, classify the context element ranges to the one set. Different ranges of a context element can be included in different context sets.

- Associate the modeling objects with the help of the meta-model depicted in Figure 5.

As mentioned in the beginning of this section, method's users that are knowledgeable in conceptual modeling can perform the analysis phase in parallel with the design phase. In that case the expected outcomes can be both models and tabular documentations, or even only one of them. 
The output of the design phase is a capability model including the business process models, goal models and context models reflecting the business idea in a holistic way. For this, the method user is provided with a notation and stencils for MicrosoftVisio (Windows) and OmniGraffle (MacOS).

\subsection{Notation Used by the LightCDD}

The LightCDD notation is illustrated in Figure 6. The method's user can either to use the CDT from the CDD suite or apply MicrosoftVisio (Win) and OmniGraffle (Mac OS) stencils supporting this notation, which can be downloaded from the following link: http://bit.ly/2jpRaOC.

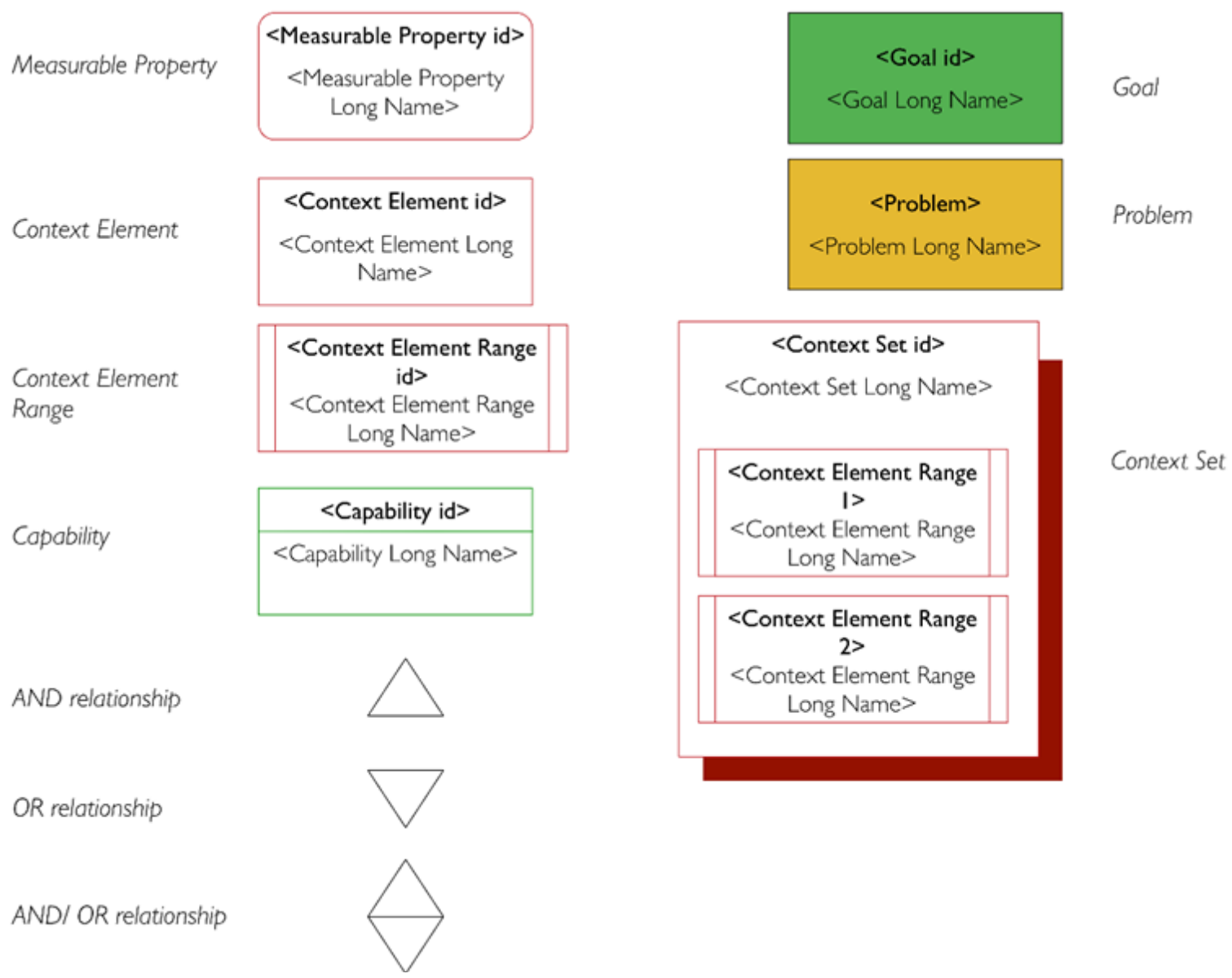

Figure 6. The LightCDD notation ${ }^{\S}$

\section{Example Case}

Let's Get Better is an online platform that provides users with a health coach in their pocket. The main motivation of the project is that an active and healthy life is the key to a well-balanced life but the people struggle adopting healthy habits, since they are busy with their work and social life. As a solution, the platform takes advantage of digital technology and offers 1:1 professional coaching through instant messaging and weekly video calls, progress tracking and peer-to-peer networks. In detail, using a special adjustment algorithm, the platform will match a user to a coach based on the user context, such as its needs, availability, and budget. Regular

${ }^{\S}$ The notation of goal modeling elements Goal and Problem is adopted from For Enterprise Modeling (4EM) approach [24]. 
communication via instant messaging and weekly video calls will provide the user with an opportunity to discuss progress, express concerns, and explore solutions, all of which will be tracked by the user goals. To further support and encourage users, a social media will allow people to share their story, gain further support, and build common-interest relationships.

The platform will be available in both website and app form. There will be a free version whereby users can interact with peer-to-peer networks, search for coaches and request free consultations. Monthly subscriptions will be offered on a tiered basis with a $€ 45, € 80$, and $€ 110$ option. Each tier will have access to different levels of professional, starting from those that are only recently qualified to those who have been practicing for $5+$ years. Coaches will be paid either $€ 20, € 30$ or $€ 40$ per hour and will spend 2 hours with each customer per month providing the business with a minimum profit margin of $8 \%$.

\subsection{Preparation Phase}

In this phase, the questions provided by the method were answered as follows (cf. Table 6), which argue for the application of the LightCDD to the use case.

Table 6. Investigating the need for the LightCDD

\begin{tabular}{|l|l|}
\hline \multicolumn{1}{|c|}{ Questions } & \multicolumn{1}{c|}{ Answers } \\
\hline $\begin{array}{l}\text { Do you plan to offer the same business for different } \\
\text { target groups and markets? }\end{array}$ & $\begin{array}{l}\text { Yes. The platform will be available in both website } \\
\text { and app. Moreover, different options for monthly } \\
\text { subscriptions are envisioned. }\end{array}$ \\
\hline $\begin{array}{l}\text { Are there are variations in the execution of business } \\
\text { ideas or certain situations for various application } \\
\text { scenarios? }\end{array}$ & $\begin{array}{l}\text { Yes. The above motivated cases will cause variations } \\
\text { in the implementation of our business idea. For } \\
\text { instance, based on their subscription type, our } \\
\text { customers will have access to the coaches with } \\
\text { varying experience levels. }\end{array}$ \\
\hline $\begin{array}{l}\text { Is your business idea is closely related to } \\
\text { digitalization and deliver ICT-based solutions? }\end{array}$ & $\begin{array}{l}\text { Yes. Let's Get Better is an online platform which } \\
\text { takes advantage of digital technology. }\end{array}$ \\
\hline $\begin{array}{l}\text { Have you used a proprietary development } \\
\text { methodology that supports your system } \\
\text { development life-cycle thoroughly. }\end{array}$ & $\begin{array}{l}\text { No. We have not used any methodology to support our } \\
\text { development life cycle. }\end{array}$ \\
\hline
\end{tabular}

\subsection{Analysis Phase}

As a first step, the entrepreneurs should specify the core proposition of the business idea, which is termed as capability in the LightCDD. For this, they list a number of implementation scenarios that may have variations in their execution. What remains same in such situations is captured as a capability.

- The experience of the coaches allocated to the users depends on the users' subscription type. For instance, users paying the lowest monthly subscription fare (Tier 1) are matched with the coaches that have a maximum of 3 years' experience. On the contrary, Tier 3 work with the most experienced coaches.

- When allocating the health coaches with users, the algorithm favors users of higher tiers. In addition to that, the service provided to the subscribers is ad-free. Non-paying users get only consultancy from a coach, during which ads are generated.

- In each condition, users should work (either train or get consultancy) with the coaches that suit best to their situation. In other words, Let's Get Better should provide an online and personalized health coaching. As such, the entrepreneurs defined this principle as their main capability. 
In the second step, important goals that should be fulfilled by implementing the business idea are listed (cf. Table 7). The goals can be associated with the problems, which may hinder their satisfaction.

Table 7. Let's Get Better Goals

\begin{tabular}{|l|l|}
\hline \multicolumn{1}{|c|}{$\begin{array}{c}\text { Problems/ } \\
\text { objectives }\end{array}$} & Description \\
\hline Problem 1 & People do not keep their promises related to weight losing \\
\hline Problem 2 & Healthy lifestyle is perceived as luxury \\
\hline Problem 3 & $\begin{array}{l}\text { The industrial solutions are not flexible enough for the people that are busy with their } \\
\text { work and social life }\end{array}$ \\
\hline Goal 1 & Let's Get Better helps them to reach a well-balanced happy life \\
\hline Goal 2 & Let's Get Better helps them to keep active in an efficient way \\
\hline Goal 3 & Let's Get Better helps them to eat and drink healthily \\
\hline Goal 4 & Let's Get Better aims to provide 1:1 professional coaching \\
\hline Goal 5 & Let's Get Better aims to provide progress tracking of training activities \\
\hline Goal 6 & Let's Get Better aims to provide coaching support through instant messaging \\
\hline Goal 7 & Let's Get Better aims to provide coaching support through video calls \\
\hline Goal 8 & Let's Get Better aims to provide progress tracking of training activities \\
\hline Goal 9 & Let's Get Better aims to calculate the user needs, availability, and budget \\
\hline Goal 10 & Let's Get Better aims to match the users and coaches based on their preferences \\
\hline Goal 11 & Let's Get Better aims to let users share experiences amongst them \\
\hline
\end{tabular}

In the third step, the key activities (A) which should be executed to realize the business idea are documented in a tabular form (cf. Table 8).

Table 8. Let's Get Better Activities

\begin{tabular}{|c|c|}
\hline Activity & Description \\
\hline A1 & $\begin{array}{l}\text { The user registers to the platform and creates a profile with the goals she wants to fulfil. Paying } \\
\text { users are walked through additional payment processes. }\end{array}$ \\
\hline A2 & Run the algorithm which matches the user with a coach. \\
\hline A3 & The coach and the user agree upon a schedule. \\
\hline A4 & The user performs the exercises and receives feedback from the coaches regarding their progress. \\
\hline A5 & $\begin{array}{l}\text { After each training session, the results are captured in the system. Then, a new iteration begins, } \\
\text { in case the goals of the user are not fulfilled. }\end{array}$ \\
\hline
\end{tabular}

The entrepreneurs became aware of the fact that the activities are defined ambiguously, which leaves room for interpretations. Moreover, they had to further specify the key activities, as they were expressed on a higher level. The following questions arised during the analysis phase.

- What exactly should A2 take as an input, when assessing the needs and profile of the user? The entrepreneurs decided that a user profile should cover the aspects of the user to be 
improved and the availability of the user during the day. Moreover, to enable a precise matching, the symptoms of the users should also be captured in the system.

- What distinguishes a paid service from a free service? The entrepreneurs decide that free service users are matched with a suitable coach, whereas they are only allowed to receive free consultancy as long as they do not upgrade the service. In other words, the activities and exercises which are exposed to the paid users will not be offered to the free users. Moreover, the progress of free users towards goals will not be tracked. Nevertheless, their goals will be documented in the system to enable a smooth upgrade.

- How can one minimize the communication costs regarding the matching (A2) and schedule arrangements (A3)? The entrepreneurs decided to capture availability and experience of the coaches in their profile. At that stage it remained unclear whether this profile can be seen by free service users.

- In A5, it was not clear what happens when the user goals are fulfilled.

To select the right workflow at the runtime, factors should be extracted from the key activity description. Also by taking the improvement needs of activities into consideration, the entrepreneurs determined the following factors.

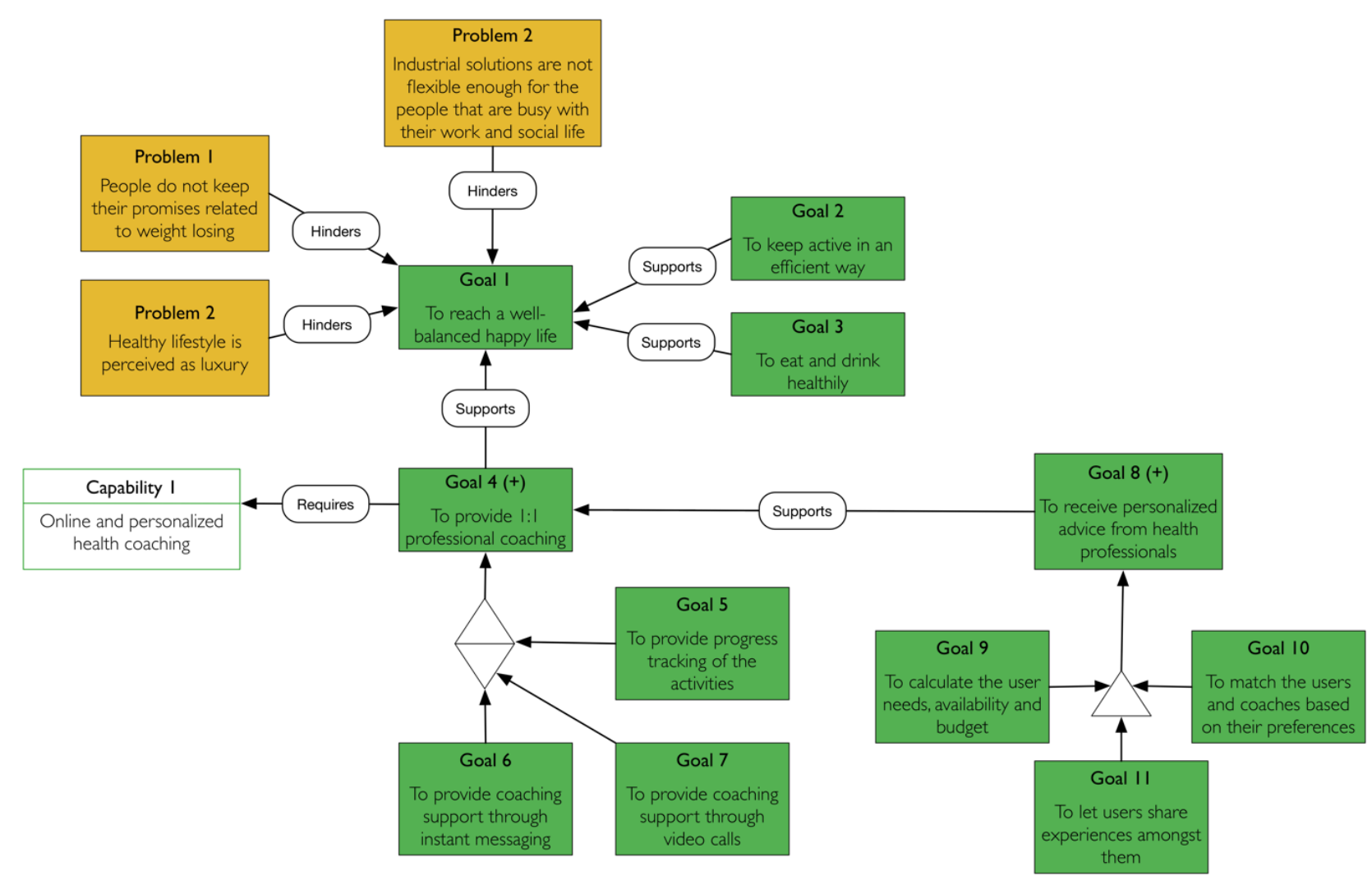

Figure 7. Core business idea and the assessment of user needs from goals perspective Design Phase

- User profile: To create successful matchings, the algorithm requires the user profile as an input. Here, three important factors determine the service provision and its quality:

- Type of the user: The service provision changes depending on the user type, thus the system has to determine whether the user pays for the service or not.

- The aspects to be improved: Before allocating a coach to a user, the app should take into consideration the aspects (fitness, diet, etc.), which a user aims to improve.

- The symptoms: If the user is not certain about the aspect she wants to be, she can select from a list of symptoms, e.g. anxiety, stress, or depression. 
- Coach profile: To enhance the preciseness, the matching algorithm should assess the current context of the coaches:

- Coach availability: In which times of the day is the respective coach available?

- Coach experience: What is the experience level of coaches? (e.g. recently qualified, practicing for $5+$ years).

The output of the analysis phase is represented with the LightCDD notation, the capability and the goals are documented in Figure 7. Note that in the initial stage, no KPIs are expressed, as they can be added in further iterations. Figure 8 illustrates the initial representation of key activities. As explained in the analysis phase, the workflow is ambiguously defined and requires further specification. According with the documented improvement needs the process model is updated (cf. the lower part of Figure 9 "Business Process"). In the last step, the contextual factors are modeled, which are represented on the left part of the capability model in Figure 9.

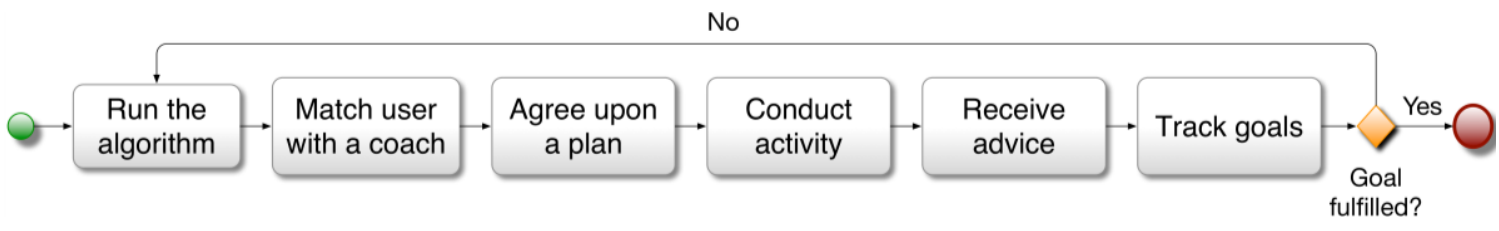

Figure 8. Initial business process model

\section{Lessons Learnt and Discussion}

Studies and analysis show that the digital economy is growing worldwide and holds huge potential for European entrepreneurs. Encouraging the entrepreneurship and innovative activities in small businesses is a key factor in economic development [7]. However, the high start-up birth rates go hand in hand with a great risk of failure.

In this article, we analysed the current problems of start-ups and focused on the lack of proper modeling methods as a threat for shutdowns. In other words, we investigated how methods and techniques for enterprise modeling could help on the development process of new ventures. We reviewed the literature and conducted a survey to know factors of failure when establishing startups. We have performed an exploratory application of CDD in two start-up projects. As a result of this experience, we found that CDD is a feasible method for start-up support. We interviewed the CEOs of the two start-up projects to know their perceptions about CDD, and how it impacted their projects. Thanks to the evidences collected during the application of CDD, the survey, the interview to the CEOs, and literature related research; we found evidences on the need to design a lightweight method for start-ups. As a result, we proposed the LightCDD, a simplified version of CDD for entrepreneurship support, which is designed based on the following principles:

- The users of LightCDD should be supplied with the information of what enterprise modeling in general is and how this could help to improve their case. Here, the specific terms regarding the capability modeling should be described. If possible, the description should be enriched with the examples from the cases.

- The LightCDD should focus on the goals, context, and processes. The pattern modeling method component is excluded, since the business idea is somewhat unique and no best practices in the application field are expected. The adjustments modeling method component is also excluded due to its strong focus on the implementation of the business idea, which is the run-time aspect.

- The prerequisites for the method application are not taken into consideration. Likewise, the stakeholder types that participate to the different phases of the CDD were excluded in this version, due to the fact that the start-up teams include smaller number of individuals.

- From the CDD tool suite, CDT seems to be the only one that the entrepreneurs can use. This is advocated by the reason that for the business design, they only need CDD tools that 
support enterprise modeling. Furthermore, the method users are provided with MicrosoftVisio (Win) and OmniGraffle (Mac OS) stencils.

We gained interesting feedback from the CEOs of the two projects in which CDD was applied, and the incubator managers. Due to entrepreneur context sensitive approach, we predict that the respondents address the ability of CDD to overcome challenges in changing situations, i.e. if it is a relatively stable environment for the start-up, such as selling of a certain product with certain features, the method might be too complex to use.

We argue that after applying the LightCDD to Let's Get Better case, the entrepreneurs gained the following benefits.

1. Exact and unambiguous specification of the business idea and eliminating the lack of realism.

Before applying the LightCDD, the entrepreneurs did not quite elaborate on the details of application's workflow. The business process illustrated in Figure 8 was rather generic and would not support the implementation of the entrepreneur business idea. After the application of the LightCDD, the workflow was detailed (cf. Figure 9), which increased the level of "realism" and thus the chances of a successful launch.

2. Assessing the suitability of the business idea by analyzing the problems of the end user as well as the goals of the entrepreneurs.

Before the LightCDD, the entrepreneurs did not document the problems of the application's users and how their business idea would solve these. Thus, they did not examine the end user needs in detail. After applying the LightCDD, the objectives became clearer, which may ensure acceptance both amongst customers and entrepreneurs (cf. Figure 7).

3. Consideration of the dynamic factors and their integration to the workflow.

End user needs are subject to change and it is already mentioned that the start-ups need to anticipate such changes to increase their chances of survival. After the LightCDD, the dynamic factors were identified and captured in a context model.

4. Clear and graphical documentation of the business idea.

After applying the LightCDD, it is now possible to represent the core business idea in graphical means. This obviously helps to communicate the idea with various stakeholders by providing a holistic view and analyze the gaps in it.

LightCDD is going to be further developed and evaluated in new start-ups. In this respect, future work is going to focus on a few aspects. First, the guidelines and best practices for BPM are going to be improved and incorporated. This requirement stems from the feedback that the application of the (Light) CDD in further entrepreneurial projects depends on such support. Second, the CDD concepts are going to be refined and simplified. Third and last, important aspects such as the revenue models, channels, key resources, and team expertise are going to be addressed, which were mentioned during the interviews, but always remained out of the CDD boundaries. This will also shed light onto the co-existence of CDD with widespread business modeling approaches such as the Business Model Canvas, hopefully covering a gap in terms of contextual analysis and how the enterprise should design flexible business processes that facilitate coping with contingencies.

Acknowledgments. This work has been supported by the Generalitat Valenciana project IDEO (PROMETEOII/2014/039); the FPI-UPV pre-doctoral grant; the European Commission Project CaaS (FP7 611351); and the ERDF structural funds. We acknowledge the participation of the DELITELABS startup school in the yes!PoEM 2015. 


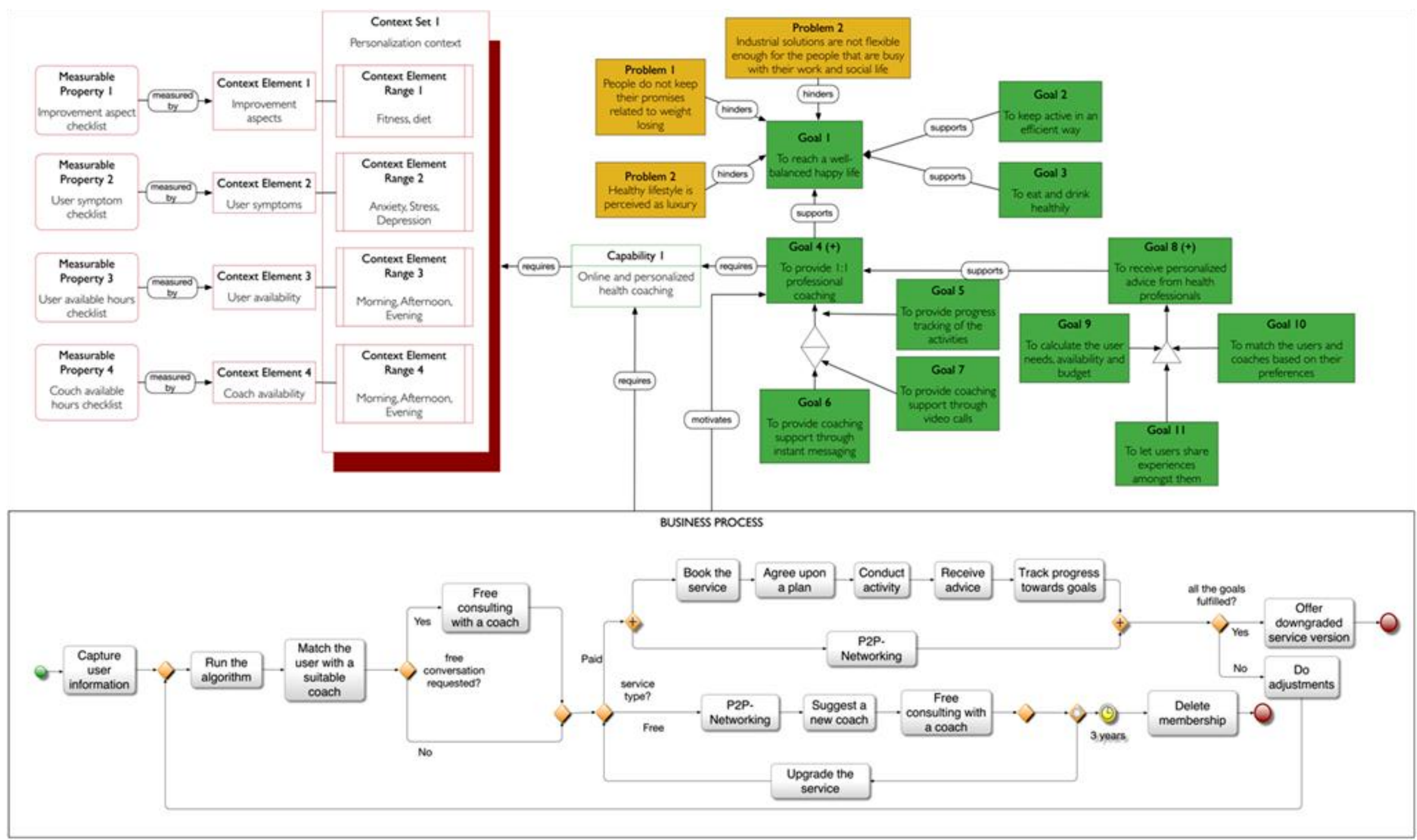

Figure 9. Capability model, including the goals, business processes, and the context 


\section{References}

[1] V. Grossmann, "Entrepreneurial Innovation and Economic Growth," Journal of Macroeconomics, vol. 31, no. 4, pp. 602-613, 2009. Available: https://doi.org/10.1016/j.jmacro.2008.12.008

[2] FRACTL, “Why Do Startups Fail?” Available: http://www.frac.t1/research/startup-failure (accessed Oct. 2016)

[3] M. Morris, M. Schindehutte and J. Allen, "The Entrepreneur's Business Model: Toward a Unified Perspective," Journal of Business Research, vol. 58, no. 6, pp. 726-735, 2005. Available: https://doi.org/10.1016/j.jbusres.2003.11.001

[4] S. Berzisa, G. Bravos, T. Cardona, U. Czubayko, S. España, J. Grabis, M. Henkel, L. Jokste, J. Kampars, H. Koç, J.-C. Kuhr, C. Llorca, P. Loucopoulos, R. Juanes Pascual, O. Pastor, K. Sandkkuhl, H. Simic, J. Stirna, F. Valverde and J. Zdravkovic, "Capability Driven Development: An Approach to Designing Digital Enterprises," Business \& Information Systems Engineering, vol. 57, no. 1, pp. 15-25, 2015. Available: https://doi.org/10.1007/s12599-014-0362-0

[5] A. Osterwalder, Y. Pigneur, T. Clark and A. Smith, "Business Model Generation: A Handbook for Visionaries, Game Changers, and Challengers," Hoboken, New Jersey: John Wiley \& Sons, 2010.

[6] R. Wieringa, "Design Science Methodology for Information Systems and Software Engineering," SpringerVerlag Berlin Heidelberg, 2014. Available: https://doi.org/10.1007/978-3-662-43839-8

[7] D. Summers, "The Economic Impact of Entrepreneurship: Setting Realistic Expectations," Academy of Entrepreneurship Journal, vol. 21, no. 2, pp. 29, 2015.

[8] Z.J. Ács, L. Serb and E. Autio, “Global Entrepreneurship Index 2016,” Available: https://thegedi.org/2016global-entrepreneurship-index/ (accessed Jan. 2016)

[9] E. Griffith, "Why Startups Fail, According to Their Founders," N.PAG, 2014. Available: http://fortune.com/2014/09/25/why-startups-fail-according-to-their-founders/

[10] N. Fallon, "Beating the Odds: 4 Steps to Startup Success," 2004. Available: http://www.foxbusiness.com/features/2014/03/13/beating-odds-4-steps-to-startup-success.html (accessed Feb. 2016)

[11] S. Chorev and A.R. Anderson, "Success in Israeli High-Tech Start-Ups; Critical Factors and Process," Technovation, vol. 26, no. 2, pp. 162-174, 2006. Available: https://doi.org/10.1016/j.technovation.2005.06.014

[12] A. Chwolka and M.G. Raigh, "The Value of Business Planning Before Start-Up - A Decision-Theoretical Perspective," Journal of Business Venturing, vol. 27, no. 3, pp. 385-399, 2012. Available: https://doi.org/10.1016/j.jbusvent.2011.01.002

[13] P. Koellinger, M. Minniti and C. Schade, ““'I Think I Can, I Think I Can”: Overconfidence and Entrepreneurial Behavior," Journal of Economic Psychology, vol. 28, no. 4, pp. 502-527, 2007. Available: https://doi.org/10.1016/j.joep.2006.11.002

[14] A. Maurya, "Running Lean: Iterate From Plan A to a Plan That Works," Beijing, Boston, Farnham: O'Reilly, 2nd edition, pp. 240, 2012.

[15] E.C. Kurucz, B.A. Colbert, F. Lüdeke-Freund, A. Upward and B. Willard, "Relational Leadership for Strategic Sustainability: Practices and Capabilities to Advance the Design and Assessment of Sustainable Business Models," Systematic Leadership towards Sustainability, vol. 140, Part 1, pp. 189-204, 2017. Available: https://doi.org/10.1016/j.jclepro.2016.03.087

[16] Board of Innovation, "Board of Innovation Business Model Kit," Available: https://www.boardofinnovation.com/ (accessed Dec. 2016)

[17] H. Koç and K. Sandkuhl, "A Business Process Based Method for Capability Modelling," in Lecture Notes in Business Information Processing, vol. 229, Perspectives in Business Informatics Research, R. Matulevicius and M. Dumas, Eds., Springer International Publishing, pp. 257-264, 2015. Available: https://doi.org/10.1007/978-3-319-21915-8_17

[18] G. Bravos, T. González, J. Grabis, M. Henkel, L. Jokste, H. Koc and J. Stirna, "Capability Modeling: Initial Experiences," in Proc. the Lecture Notes in Business Information Processing, B. Johansson, B. Andersson and N. Holmberg, Eds., vol. 194, Perspectives in business informatics research: 13th International Conference, BIR 2014, Lund, Sweden, September 22-24, 2014. Cham: Springer, pp. 1-14, 2014. Available: https://doi.org/10.1007/978-3-319-11370-8_1 
[19] S. España, J. Grabis, M. Henkel, H. Koç, K. Sandkuhl, J. Stirna and J. Zdravkovic, "Strategies for Capability Modelling: Analysis Based on Initial Experiences," in Lecture Notes in Business Information Processing, vol. 215, Advanced Information Systems Engineering Workshops, A. Persson and J. Stirna, Eds., Springer International Publishing, 2015, pp. 40-52. Available: https://doi.org/10.1007/978-3-319-19243-7_4

[20] J. Grabis, M. Henkel, J. Kampars, H. Koç, K. Sandkuhl, D. Stamer, J. Stirna, F. Valverde and J. Zdravkovic, "Deliverable 5.3: The Final Version of Capability Driven Development Methodology: CaaS - Capability as a Service in Digital Eenterprises, Collaborative Project Number 611351," Stockholm University, Department of Computer and Systems Sciences; Universitat Politècnica de València, Centro de Investigación PROS; Riga Technical University; University of Rostock, 2016.

[21] P. Johannesson and E. Perjons, “An Introduction to Design Science," 1st edition, Cham: Springer, 2014. Available: https://doi.org/10.1007/978-3-319-10632-8

[22] A. Voinov and E.J.B. Gaddis, "Lessons for Successful Participatory Watershed Modeling: A Perspective From Modeling Practitioners," Ecological Modelling, vol. 216, no. 2, pp. 197-207, 2008. Available: https://doi.org/10.1016/j.ecolmodel.2008.03.010

[23] Object Management Group, "Business Process Model and Notation," v2.0, 2011. Available: http://www.omg.org/spec/BPMN/2.0/PDF (accessed Jan. 2017)

[24] K. Sandkuhl, J. Stirna, A. Persson and M. Wißotzki, "Enterprise Modeling: Tackling Business Challenges With the 4EM Method," Enterprise Modeling, 1st edition, Springer-Verlag Berlin Heidelberg, 2014. Available: https://doi.org/10.1007/978-3-662-43725-4

[25] I. van de Weerd and S. Brinkkemper, "Meta-modeling for situational analysis and design methods," Handbook of research on modern systems analysis and design technologies and applications. M. R. Syed and S. N. Syed (ed.), IGI Global, pp. 35-54, 2009. 